\title{
Reduction of the cetrorelix dose in a multiple-dose antagonist protocol and its impact on pregnancy rate and affordability: A randomized controlled multicenter study
}

\author{
Ayman S. Dawood, Adel Algergawy, Ahmed Elhalwagy \\ Department of Obstetrics and Gynaecology, Faculty of Medicine, Tanta University, Tanta, Egypt
}

Objective: To determine whether reducing the cetrorelix dose in the antagonist protocol to $0.125 \mathrm{mg}$ had any deleterious effects on follicular development, the number and quality of retrieved oocytes, or the number of embryos, and to characterize its effects on the affordability of assisted reproductive technology.

Methods: This randomized controlled study was conducted at the Fertility Unit of Tanta Educational Hospital of Tanta University, the Egyptian Consultants' Fertility Center, and the Qurrat Aien Fertility Center, from January 1 to June 30, 2017. Patients' demographic data, stimulation protocol, costs, pregnancy rate, and complications were recorded. Patients were randomly allocated into two groups: group I $(n=61)$ received $0.125 \mathrm{mg}$ of cetrorelix (the study group), and group II ( $n=62)$ received $0.25 \mathrm{mg}$ of cetrorelix (the control group).

Results: The demographic data were comparable regarding age, parity, duration of infertility, and body mass index. The dose of recombinant follicle-stimulating hormone units required was 2,350.43 $\pm 150.76 \mathrm{IU}$ in group I and 2,366.25 $\pm 140.34 \mathrm{IU}$ in group II, which was not a significant difference $(p=0.548)$. The duration of stimulation, number of retrieved oocytes, and number of developed embryos were not significantly different between the groups. The clinical and ongoing pregnancy rates likewise did not significantly differ. The cost of intracytoplasmic sperm injection per cycle was significantly lower in group I than in group II (US $\$ 494.66 \pm 4.079$ vs. US $\$ 649.677 \pm 43.637$ ).

Conclusion: Reduction of the cetrorelix dose in the antagonist protocol was not associated with any significant difference either in the number of oocytes retrieved or in the pregnancy rate. Moreover, it was more economically feasible for patients in a low-resource country.

Keywords: Antagonist; Assisted reproductive techniques; Gonadotropins

\section{Introduction}

The antagonist protocol involves controlled ovarian hyperstimulation $(\mathrm{COH})$ starting from the third day of menses until the follicles reach $14 \mathrm{~mm}$, at which point a gonadotropin-releasing hormone

Received: Sep 14, 2017· Revised: Nov 15, 2017 · Accepted: Nov 29, 2017 Corresponding author: Ayman S. Dawood

Department of Obstetrics and Gynaecology, Faculty of Medicine, Tanta University, Tanta 31111, Egypt

Tel:+20-1225880071 E-mail:ayman.dawood@med.tanta.edu.eg

This is an Open Access article distributed under the terms of the Creative Commons Attribution Non-Commercial License (http://creativecommons.org/licenses/by-nc/4.0/) which permits unrestricted non-commercial use, distribution, and reproduction in any medium, provided the original work is properly cited.
$(\mathrm{GnRH})$ antagonist is given daily to prevent the luteinizing hormone (LH) surge. It is used mainly in older women, poor responders, and women with polycystic ovary syndrome [1].

The introduction of $\mathrm{GnRH}$ antagonists (GnRH-ant) as part of assisted reproductive technology to prevent the LH surge provided a new way of making in vitro fertilization (IVF) safer and friendlier. Unlike the indirect pituitary suppression induced by $\mathrm{GnRH}$ agonists (GnRH-a), $\mathrm{GnRH}$-ant administration causes the immediate and dose-related inhibition of gonadotropin release by competitive occupancy of the $\mathrm{GnRH}$ receptors in the pituitary gland [2,3].

In the multiple-dose protocol, the GnRH-ant is administered continuously until the day of human chorionic gonadotropin ( $\mathrm{hCG}$ ) ad- 
ministration, and the minimal effective dose to prevent the occurrence of a premature $\mathrm{LH}$ rise was identified as $0.25 \mathrm{mg}$ of cetrorelix $[2,4]$.

The optimal levels of endogenous LH in GnRH-ant cycles are still a matter of debate. It may be assumed that the deep suppression of LH secretion induced by GnRH-a administration is likely to be detrimental for the follicle-oocyte complex. Low residual LH concentrations and impaired estradiol $\left(E_{2}\right)$ secretion with increasing antagonist doses were indeed associated with low implantation rates [5].

In contrast, a trend towards lower pregnancy rates was observed in patients with $\mathrm{LH}$ deficiency, documented by a low $\mathrm{E}_{2}$-to-oocyte ratio, which could be explained by the endometrial impact of low LH levels [6]. On the basis of these observations, the possibility of LH supplementation in $\mathrm{GnRH}$-ant regimens was examined. Data from two randomized controlled trials showed that the addition of $75 \mathrm{IU}$ of recombinant LH to recombinant FSH at GnRH-ant initiation, or starting at the initiation of stimulation, did not appear to increase pregnancy rates [7]. Similarly, no improvement in pregnancy rates was found after increasing the dose of gonadotropins by $75 \mathrm{IU}$ at $\mathrm{GnRH}$-ant initiation [8]. Neither study showed any evidence that low endogenous LH levels after $\mathrm{GnRH}$-ant initiation were associated with a decreased probability of pregnancy in IVF cycles [8].

In a third study conducted by Baruffi et al. [9], a meta-analysis of five randomized controlled trials, significantly higher serum $\mathrm{E}_{2}$ concentrations and greater numbers of MII oocytes were observed in $\mathrm{GnRH}$-ant cycles supplemented with LH, suggesting that LH may prevent any decrease in $\mathrm{E}_{2}$ levels after antagonist administration, even if there was no significant difference in the implantation and pregnancy rates.

Chang et al. [10] conducted a prospective randomized controlled trial in which a total of 58 women with a body weight of $40-50 \mathrm{~kg}$ were allocated into two groups. The patients in group $1(n=28)$ were given a fixed cetrorelix dose of $0.2 \mathrm{mg} /$ day, and the patients in group $2(n=30)$ were given a fixed cetrorelix dose of $0.15 \mathrm{mg} /$ day. The authors found that there were no statistically significant differences between the two groups in terms of the clinical pregnancy rate, gonadotropin dose, or incidence of ovarian hyperstimulation syndrome (OHSS).

In this study, a reduced cetrorelix dose $(0.125 \mathrm{mg})$ was compared to the full dose $(0.25 \mathrm{mg})$ in terms of the required dose of gonadotropins, pregnancy rate, and costs.

\section{Methods}

\section{Study design and setting}

This study was a non-blind, prospective, double-armed, randomized clinical trial. The study was conducted at three centers, the Edu- cational Hospital Fertility Unit of Tanta University, the Egyptian Consultants' Fertility Center in Tanta, and the Qurrat Aien fertility Center in El-Mahalla Al-Kubra, from January 1 to June 30, 2017.

\section{Eligibility}

The enrolled patients $(n=223)$ were assessed for eligibility according to the following inclusion and exclusion criteria. The inclusion criteria were: (1) age <35 years, (2) women with polycystic ovaries, (3) $\mathrm{FSH}<10 \mathrm{IU} / \mathrm{mL}$, (4) antral follicle count $>3$, (5) anti-Müllerian hormone (AMH) levels $\geq 1 \mathrm{ng} / \mathrm{mL}$, (6) body mass index $\leq 26 \mathrm{~kg} / \mathrm{m}^{2}$, and (7) the presence of both ovaries. The exclusion criteria were a history of endometriosis, a history of more than two implantation failures, and a history of previous ovarian surgery.

\section{Randomization and allocation}

The 155 eligible women were randomly allocated into two groups. Group I ( $n=78$ ) received $0.125 \mathrm{mg}$ of cetrorelix (the study group) and group II ( $n=77$ ) received $0.25 \mathrm{mg}$ of cetrorelix (the control group). Randomization was performed by a computer program, with random numbers denoting the treatment modality. These numbers were placed in closed envelopes, and allocation was done according to these numbers. The envelope openers did not change participants' allocation.

\section{Interventions}

\section{1) Stimulation protocol}

All participants were treated with a $\mathrm{GnRH}$-ant protocol. The patients received recombinant human FSH (150 IU subcutaneously; Gonal-F, Merck Serono, Modugno, Italy) for 5 days. Serial transvaginal sonography was performed. When a mature follicle ( $\geq 14 \mathrm{~mm}$ ) was detected, a cetrorelix $(0.125 \mathrm{mg} /$ day, subcutaneously; Cetrotide, Merck Serono) was injected in group I, while $0.25 \mathrm{mg} /$ day of cetrorelix was injected subcutaneously in group II. Triggering was commenced with 10,000 IU of hCG (Choriomon; IBSA, Washington, DC, USA) through an intramuscular injection when at least two follicles with a mean diameter of $18 \mathrm{~mm}$ were observed.

\section{2) Ovum pickup}

Transvaginal egg retrieval was done under sedation by $0.1-0.15$ $\mathrm{mg} / \mathrm{kg} / \mathrm{min}$ of intravenous propofol for 3-5 minutes; it was titrated to the desired clinical effect and administered as a slow infusion or a slow intravenous injection. Ovum pickup was commenced after 36 hours for all patients.

\section{3) Luteal support}

Progesterone (vaginal suppositories, Cyclogest 400 mg; Actavis, Barnstaple, UK) was administered twice daily for luteal phase sup- 
port. Luteal support started after ovum pickup and continued until evidence of cardiac pulsation was detected by ultrasonography.

\section{4) Embryo transfer}

On day 5 after oocyte retrieval, 2-3 embryos were transferred using a Labotect catheter (Labotect, Gottingen, Germany) in all patients. Serum $\beta$-hCG was assessed on day 14 after embryo transfer.

As the transfer was done on day 5; embryos were graded according to the Gardner system [11]. Good-quality embryos (grades 3-4) were defined as those in which the blastocoel completely filled the embryo, the inner cell mass was loosely grouped with several cells (grade B), and the trophectoderm had very few cells, forming a loose epithelium (grade B). Poor-quality embryos (grade 1 or 2) were defined as those with a quality lower than 3BB on day 5 [12].

\section{Study outcomes and definitions}

The primary outcomes included the number of oocytes retrieved, the number of developed embryos, the progesterone level on the triggering day, and the clinical/ongoing pregnancy rates. The secondary outcomes were the cost of the cycle and the occurrence of complications.

The clinical pregnancy rate was defined as evidence of pregnancy by clinical (foetal heartbeat) or ultrasound parameters (ultrasound visualization of a gestational sac, embryonic pole with heartbeat). The ongoing pregnancy rate was defined as the number of pregnancies that continued beyond 12 weeks of gestation.

\section{Ethical approval and trial registration}

The study was approved by the local Institutional Review Board (the ethics committee) of Tanta University (No. 31241/12/16) and was registered in the UMIN Clinical Trial Registry (ID: UMIN000027193).

\section{Statistical methods}

\section{1) Sample size calculation}

The sample was calculated using Epi Info ver. 7.0 (Centers for Disease Control and Prevention, Atlanta, GA, USA); $\mathrm{HO}$ was postulated to indicate that the reduced cetrorelix dose would have the same effect as the full dose. Assuming a confidence level of $95 \%$, a confidence interval of 5 , and a percentage of $50 \%$, the estimated sample was 155 patients.

\section{2) Statistical analysis}

All statistical analyses were carried out using SPSS ver. 18.0 (SPSS Inc., Chicago, IL, USA). The normality of the data distribution was checked. The mean \pm standard deviation was calculated for the descriptive analysis. The independent $t$-test was used to analyse differences. Statistical significance was considered to be indicated by $p$-values $<0.05$. According to the power analysis, the power of the study was 0.8 and a was 0.05 .

\section{Results}

The patients enrolled at the three centers $(n=223)$ were assessed for eligibility, and 68 patients were excluded either because they did not

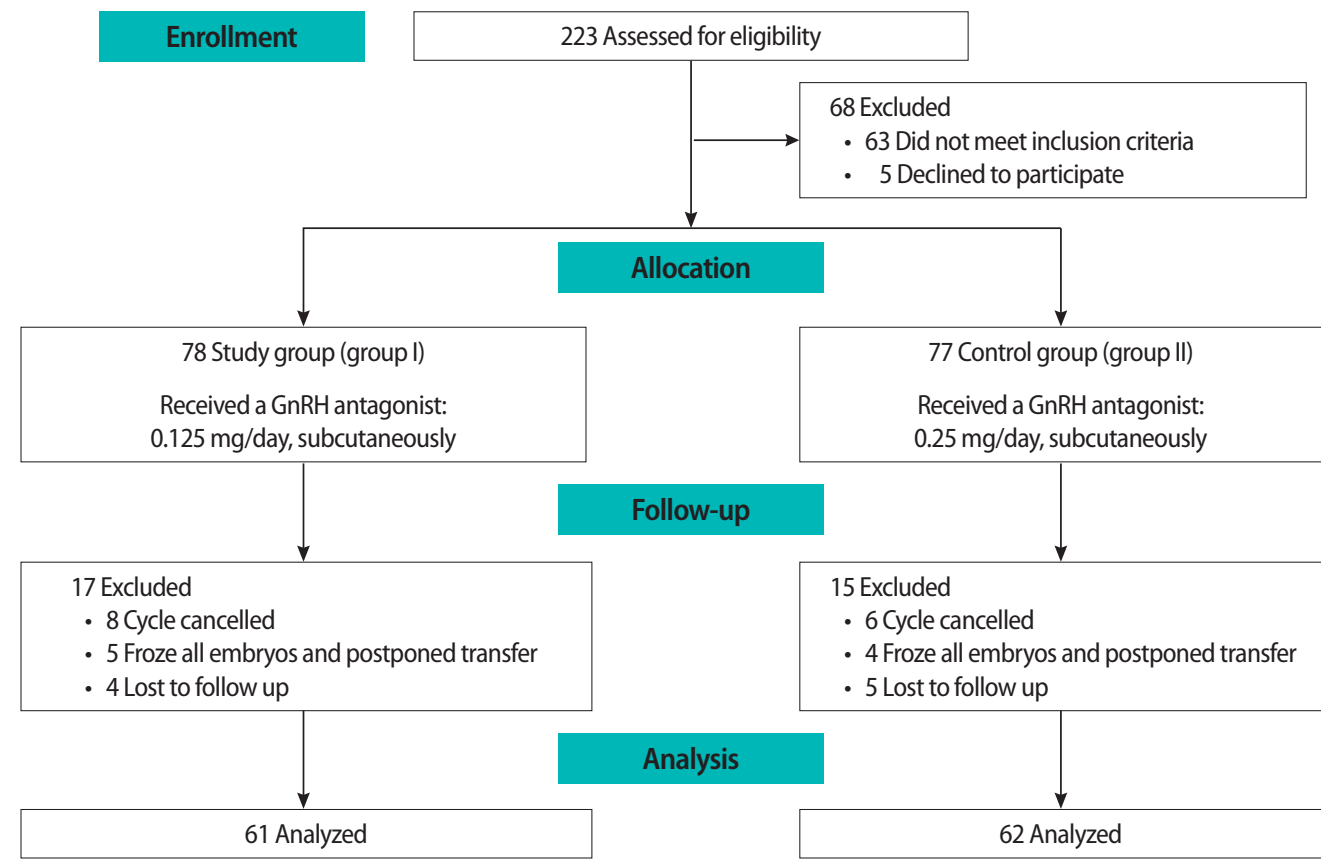

Figure 1. Flowchart of included patients and their management options. $\mathrm{GnRH}$, gonadotropin-releasing hormone. 
meet the inclusion criteria $(n=63)$ or declined to participate $(n=5)$. The eligible patients $(n=155)$ were allocated into two groups: the study group (group I, $n=78$ ) and the control group (group II, $n=77$ ). Subsequently, 17 patients were excluded from group I and 15 patients from group II for other reasons, as shown in the flowchart in Figure 1.

The demographic characteristics, type and duration of infertility, and hormonal profile including day $3(\mathrm{AMH}, \mathrm{FSH})$ were comparable in both groups. The causes of infertility in the participants are presented in Table 1.

The cycle characteristics in both groups are shown in Table 2. The mean dose of recombinant FSH was 2,350.43 \pm 150.76 IU in group I and $2,366.25 \pm 140.34 \mathrm{IU}$ in group II, with no significant difference

Table 1. Basic characteristics of the patients enrolled in the study and control groups

\begin{tabular}{lccc}
\hline Parameter & Study group $(\mathrm{n}=61)$ & Control group $(\mathrm{n}=62)$ & $p$-value \\
\hline Age $(\mathrm{yr})$ & $24.8-32.9(27.21 \pm 2.33)$ & $23.7-31.11(26.79 \pm 2.50)$ & 0.337 \\
Parity & $0-2(0.885 \pm 0.839)$ & $0-2(1.210 \pm 2.818)$ & 0.389 \\
BMI $\left(\mathrm{kg} / \mathrm{m}^{2}\right)$ & $23.40-27.10(25.08 \pm 1.63)$ & $22.11-24.59(25.33 \pm 1.34)$ & 0.354 \\
Duration of infertility (yr) & $3.2-7.8(5.24 \pm 1.80)$ & $4.3-6.6(5.33 \pm 2.01)$ & 0.794 \\
Type of infertility & & & 0.605 \\
Primary & $43(70.49)$ & $41(66.13)$ & 0.605 \\
Secondary & $18(29.51)$ & $21(33.87)$ & 0.842 \\
Cause of infertility & $10(16.39)$ & $11(17.74)$ & 0.787 \\
Polycystic ovary syndrome & $13(21.31)$ & $12(19.35)$ & 0.875 \\
Bilateral tubal block & $11(18.03)$ & $10(19.13)$ & 0.780 \\
Male factor & $27(44.26)$ & $29(46.77)$ & \\
Unexplained & & & \\
Hormonal profile & $3.22 \pm 1.30$ & $3.62 \pm 1.70$ & 0.146 \\
AMH (ng/mL) & $6.54 \pm 1.39$ & $6.49 \pm 1.82$ & 0.865 \\
FSH (IU/mL) & & \\
\hline
\end{tabular}

Values are presented as range (mean \pm standard deviation) or number (\%).

$\mathrm{BMI}$, body mass index; $\mathrm{AMH}$, anti-Müllerian hormone; $\mathrm{FSH}$, follicle-stimulating hormone.

a) On day 3.

Table 2. Cycle characteristics and ICSI outcomes in the study and control groups

\begin{tabular}{lccc}
\hline Parameter & Study group $(\mathrm{n}=61)$ & Control group $(\mathrm{n}=62)$ & $p$-value \\
\hline Dose of $\mathrm{rFSH}(\mathrm{IU})$ & $2,025-2,362.5(2,350.43 \pm 150.76)$ & $1,800-2,625(2,366.25 \pm 140.34)$ & 0.548 \\
Duration of stimulation (day) & $6-9(7.78 \pm 0.967)$ & $6-10(7.64 \pm 0.990)$ & 0.429 \\
LH level (IU/mL) & $3.5 \pm 1.44$ & $3.3 \pm 1.88$ & 0.509 \\
Progesterone $(\mathrm{ng} / \mathrm{mL})^{\mathrm{a})}$ & $0.82 \pm 0.12$ & $2,17 \pm 0.10$ & 0.013 \\
Estradiol (pg/mL) & $2,169.39 \pm 131.45$ & $6-13(9.613 \pm 2.051)$ & 0.276 \\
No. of retrieved oocytes & $8-12(10.132 \pm 1.340)$ & & 0.099 \\
Quality of oocytes & & $5-6(5.466 \pm 0.504)$ & 0.581 \\
Mature & $4-5(4.416 \pm 0.498)$ & $2-3(2.514 \pm 0.503)$ & 0.644 \\
Immature & $2-3(2.472 \pm 0.503)$ & $7-9(7.174 \pm 0.638)$ & 0.761 \\
No. of developed embryos & $6-8(7.213 \pm 0.777)$ & & \\
Quality of developed embryos & & $2-4(2.145 \pm 0.355)$ & $1-2(1.545 \pm 0.482)$ \\
$\quad$ Good quality & $2-3(2.229 \pm 0.424)$ & 79.23 & 0.235 \\
Poor quality & $1-2(1.453 \pm 0.501)$ & 28.8 & 0.301 \\
Fertilization rate (\%) & 76.67 & $12.9-17.3(14.638 \pm 1.674)$ & 0.733 \\
Implantation rate (\%) & 26.4 & $30(48.34)$ & 0.766 \\
Endometrial thickness (mm) & $13.6-18.2(15.212 \pm 1.793)$ & $25(40.32)$ & 0.068 \\
Clinical pregnancy rate & $29(47.5)$ & $560-700(649.677 \pm 43.637)$ & 0.926 \\
Ongoing pregnancy rate & $22(36.07)$ & & 0.629 \\
Cost of one ICSI trial (US \$) & $490-500(494.66 \pm 4.079)$ & 0.001 &
\end{tabular}

Values are presented as range (mean \pm standard deviation) or number (\%).

ICSI, intracytoplasmic sperm injection; rFSH, recombinant follicle-stimulating hormone; LH, luteinizing hormone.

a) On the day of triggering. 
Table 3. Complications in the study and control groups

\begin{tabular}{lccc}
\hline Parameter & $\begin{array}{c}\text { Study group } \\
(\mathrm{n}=61)\end{array}$ & $\begin{array}{c}\text { Control group } \\
(\mathrm{n}=62)\end{array}$ & $p$-value \\
\hline $\begin{array}{l}\text { Premature LH surge on } \\
\text { triggering day }(>10 \mathrm{IU} / \mathrm{mL})\end{array}$ & $3 / 61(4.9)$ & $2 / 62(3.23)$ & 0.640 \\
OHSS & None & None & - \\
Cycle cancellation & $8 / 61(13.11)$ & $6 / 62(9.68)$ & 0.551 \\
Multiple pregnancy rate & $5 / 29(17.24)$ & $7 / 30(23.33)$ & 0.565 \\
Abortion rate & $8 / 29(27.59)$ & $6 / 30(20.00)$ & 0.497 \\
\hline
\end{tabular}

Values are presented as number (\%).

$\mathrm{LH}$, luteinizing hormone; OHSS, ovarian hyperstimulation syndrome.

between the groups $(p=0.548)$. The duration of stimulation (days) was $7.78 \pm 0.967$ vs. $7.64 \pm 0.990$ in the study and control groups, respectively $(p=0.429)$. LH levels on the triggering day of $>10 \mathrm{IU} / \mathrm{mL}$ were observed in three cases (4.9\%) in the study group and in two cases (3.23\%) in the control group. The number of oocytes retrieved in both groups was nearly the same: $10.132 \pm 1.340$ in group I and $9.613 \pm 2.051$ in group II $(p=0.099)$. There was a nonsignificant difference in the number of developed embryos $(7.213 \pm 0.777 \mathrm{vs}$. $7.174 \pm 0.638$ in groups I and II, respectively), as shown in Table 2 .

The endometrial thickness was $15.212 \pm 1.793 \mathrm{~mm}$ in group I and $14.638 \pm 1.674 \mathrm{~mm}$ in group II $(p=0.068)$. The clinical pregnancy rate was similar in both groups: 29 of 61 (47.5\%) in group I and 30 of 62 (48.34\%) in group II ( $p=0.926)$. The ongoing pregnancy rates were also comparable in both groups (22/61 [36.07\%] and 25/62 [40.32\%] in groups I and II, respectively), with a $p$-value of 0.629 . The main difference was found in the financial cost of each cycle; the cost per cycle was significantly lower in group I than in group II (US $\$ 494.66 \pm$ 4.079 vs. US $\$ 649.677 \pm 43.637$, respectively), with a $p$-value of $<0.001$, as shown in Table 2 .

There were no reported cases of OHSS in either group. Other complications are reported in Table 3. Fourteen patients in the current study were cancelled due to a poor response $(n=6)$, a premature $\mathrm{LH}$ surge $(n=5)$, or financial causes $(n=3)$.

\section{Discussion}

Currently, GnRH-ant protocols are widely used to suppress endogenous gonadotropins to prevent a premature $\mathrm{LH}$ rise during $\mathrm{COH}$ in IVF-embryo transfer cycles. In the past, $\mathrm{GnRH}$-ant was restricted to certain populations, such as older patients and those with previous poor outcomes [8].

$\mathrm{GnRH}$-ant compounds were developed at the same time as GnRHa drugs, but they were associated with a high incidence of histamine release following injection. The introduction of third- and fourthgeneration GnRH-ant drugs (cetrorelix and ganirelix) minimized histamine release, enabling $\mathrm{GnRH}$-ant to be used in multiple-dose regi- mens in women undergoing $\mathrm{COH}$ [13]. With GnRH-ant, rapid suppression of the pituitary gland takes place, as well as a rapid recovery of pituitary function due to the short elimination half-life, so the degree of suppression can be adjusted by changing the dose of $\mathrm{GnRH}$ ant [14].

$\mathrm{GnRH}$-ant protocols have clinical pregnancy and live birth rates comparable to $\mathrm{GnRH}$-a long protocols, which have been the standard stimulation protocols for many years. $\mathrm{GnRH}$-ant protocols have less likelihood of OHSS and a shorter gonadotropin stimulation period (less cost), making them cost-effective. Moreover, with GnRH-ant, triggering with $\mathrm{GnRH}$-a could be used with minimal OHSS incidence. Al-Inany et al. [14] found that the use of GnRH-ant has moderatequality evidence compared with long-course $\mathrm{GnRH}$ protocols.

Many studies have tried to determine the most effective dosage of cetrorelix acetate to prevent a premature LH surge [15-17]. Albano et al. [3] compared daily doses of $0.5,0.25$, and $0.1 \mathrm{mg}$ of cetrorelix in women undergoing IVF. They found that dosages of both 0.5 and $0.25 \mathrm{mg}$ prevented a LH surge, while a premature $\mathrm{LH}$ surge was observed in two of the seven patients who received a dose of $0.1 \mathrm{mg}$.

Similarly, a multicenter study was performed on 333 women who received six different dosages of ganirelix $(0.0625,0.125,0.25,0.5,1.0$, and $2.0 \mathrm{mg} / 0.5 \mathrm{~mL}$ ) administered once daily by a subcutaneous injection. They found that serum concentrations of ganirelix increased in a linear manner, whereas serum $\mathrm{LH}$ and the increase of $\mathrm{E}_{2}$ fell with increasing ganirelix doses. During ganirelix treatment, serum LH concentrations $\geq 10 \mathrm{IU} / \mathrm{mL}$ were observed in the lowest dose groups, with incidences of $16 \%(0.0625 \mathrm{mg}), 9 \%(0.125 \mathrm{mg})$, and $1.4 \%(0.25$ $\mathrm{mg}$ ). On the day of $\mathrm{hCG}$ administration, the number of follicles measuring $\geq 11$, $\geq 15$, and $\geq 17 \mathrm{~mm}$ was similar across the six dose groups, whereas serum $\mathrm{E}_{2}$ concentrations were highest in the 0.0625 $\mathrm{mg}$ group $(1,475 \mathrm{pg} / \mathrm{mL})$ and lowest in the $2 \mathrm{mg}$ group $(430 \mathrm{pg} / \mathrm{mL}$ ) [4].

In the present study, the fertilization method in all cases was intracytoplasmic sperm injection (ICSI); a single fertilization method was used to avoid bias and fallacious conclusions. Although ICSI is more expensive than IVF, it was highly accepted by our study population and commonly requested. Subjects excluded from the study for financial reasons underwent IVF to complete their trial, but were not added to the population analysed in this study.

In the present study, reducing the $\mathrm{GnRH}$-ant dosage from 0.25 to $0.125 \mathrm{mg}$ was found to lead to the same clinical and ongoing pregnancy rates. This reduced dose of GnRH-ant was associated with a significant reduction in cost, from US $\$ 618.75$ for the 0.25 -mg protocol to US $\$ 499.34$ for the 0.125 -mg protocol $(p=0.003)$. There were no reported cases of OHSS, as shown in Tables 2 and 3.

Huang et al. [18] compared a reduced cetrorelix dose $(0.125 \mathrm{mg})$ to $\mathrm{GnRH}-\mathrm{a}$ in 120 unselected women. They found that the primary and 
secondary outcomes were comparable in both groups. Moreover, a shorter duration of stimulation, a lower dosage of recombinant FSH, and a thinner endometrium on the day of triggering were all observed in the $\mathrm{GnRH}$-ant group. They concluded that the reduced cetrorelix dose $(0.125 \mathrm{mg})$ was effective for these unselected patients during IVF-embryo transfer.

Another study was performed in Asian women, as they weigh less than Caucasian women; therefore, the researchers suggested that the GnRH-ant dosage should be adjusted. In that series, it was concluded that a lower dose ( $0.2 \mathrm{mg}$ daily dosage of cetrorelix) for Asians with a lower body weight $(<50 \mathrm{~kg}$ ) should be considered. They noted that $0.15 \mathrm{mg}$ of cetrorelix daily was not suitable for LH suppression, and that the clinical pregnancy/implantation rates in the $0.2 \mathrm{mg}$ group were higher than in the $0.15 \mathrm{mg}$ group. They also noted that $0.2 \mathrm{mg}$ of cetrorelix appeared to have a comparable pregnancy rate (around 30\%) to that of $0.25 \mathrm{mg}$ of cetrorelix. The LH surge risk for the patients who received the $0.2 \mathrm{mg}$ daily dose was as low as those who received $0.25 \mathrm{mg}$ [10].

A reduction of the cetrorelix dose by half in an ICSI antagonist protocol was not associated with any significant differences in the number of oocytes retrieved or in the pregnancy rate. Moreover, it was more economically feasible for patients in a low-resource country.

\section{Conflict of interest}

No potential conflict of interest relevant to this article was reported.

\section{Acknowledgments}

We want to thank all the nursing and statistics staff at the Egyptian Consultants' Center and the Qurrat Aien Center for their tremendously useful help and cooperation in data collection.

\section{References}

1. Humaidan P, Quartarolo J, Papanikolaou EG. Preventing ovarian hyperstimulation syndrome: guidance for the clinician. Fertil Steril 2010;94:389-400.

2. Huirne JA, Homburg R, Lambalk CB. Are GnRH antagonists comparable to agonists for use in IVF? Hum Reprod 2007;22:280513.

3. Albano C, Smitz J, Camus M, RiethmUller-Winzen H, Van Steirteghem A, Devroey P. Comparison of different doses of gonadotropin-releasing hormone antagonist cetrorelix during controlled ovarian hyperstimulation. Fertil Steril 1997;67:917-22.

4. A double-blind, randomized, dose-finding study to assess the efficacy of the gonadotrophin-releasing hormone antagonist ganirelix (Org 37462) to prevent premature luteinizing hormone surges in women undergoing ovarian stimulation with recombinant follicle stimulating hormone (Puregon). The ganirelix dosefinding study group. Hum Reprod 1998;13:3023-31.

5. Olivennes F, Belaisch-Allart J, Emperaire JC, Dechaud H, Alvarez S, Moreau L, et al. Prospective, randomized, controlled study of in vitro fertilization-embryo transfer with a single dose of a luteinizing hormone-releasing hormone ( $\mathrm{LH}-\mathrm{RH})$ antagonist (cetrorelix) or a depot formula of an LH-RH agonist (triptorelin). Fertil Steril 2000;73:314-20.

6. Sbracia M, Colabianchi J, Giallonardo A, Giannini P, Piscitelli C, Morgia $\mathrm{F}$, et al. Cetrorelix protocol versus gonadotropin-releasing hormone analog suppression long protocol for superovulation in intracytoplasmic sperm injection patients older than 40. Fertil Steril 2009;91:1842-7.

7. Bodri D, Sunkara SK, Coomarasamy A. Gonadotropin-releasing hormone agonists versus antagonists for controlled ovarian hyperstimulation in oocyte donors: a systematic review and metaanalysis. Fertil Steril 2011;95:164-9.

8. Itskovitz-Eldor J, Kol S, Mannaerts B. Use of a single bolus of GnRH agonist triptorelin to trigger ovulation after $\mathrm{GnRH}$ antagonist ganirelix treatment in women undergoing ovarian stimulation for assisted reproduction, with special reference to the prevention of ovarian hyperstimulation syndrome: preliminary report: short communication. Hum Reprod 2000;15:1965-8.

9. Baruffi RL, Mauri AL, Petersen CG, Felipe V, Martins AM, Cornicelli $J$, et al. Recombinant LH supplementation to recombinant FSH during induced ovarian stimulation in the $\mathrm{GnRH}$-antagonist protocol: a meta-analysis. Reprod Biomed Online 2007;14:14-25.

10. Chang YL, Hsieh YY, Tsai HD. Preliminary report on the effect of a lower dose of gonadotropin-releasing hormone antagonist (cetrorelix) on ovarian hyperstimulation in lower-weight Asian women. Taiwan J Obstet Gynecol 2006;45:317-20.

11. Gardner DK, Lane M, Stevens J, Schlenker T, Schoolcraft WB. Blastocyst score affects implantation and pregnancy outcome: towards a single blastocyst transfer. Fertil Steril 2000;73:1155-8.

12. Wintner EM, Hershko-Klement A, Tzadikevitch K, Ghetler Y, Gonen $\mathrm{O}$, Wintner $\mathrm{O}$, et al. Does the transfer of a poor quality embryo together with a good quality embryo affect the in vitro fertilization (IVF) outcome? J Ovarian Res 2017;10:2.

13. Griesinger G, Felberbaum RE, Schultze-Mosgau A, Diedrich K. Gonadotropin-releasing hormone antagonists for assisted reproductive techniques: are there clinical differences between agents? Drugs 2004;64:563-75.

14. Al-Inany HG, Youssef MA, Ayeleke RO, Brown J, Lam WS, Broekmans FJ. Gonadotrophin-releasing hormone antagonists for assisted reproductive technology. Cochrane Database Syst Rev 
2016;4:CD001750.

15. Lee TH, Wu MY, Chen HF, Chen MJ, Ho HN, Yang YS. Ovarian response and follicular development for single-dose and multipledose protocols for gonadotropin-releasing hormone antagonist administration. Fertil Steril 2005;83:1700-7.

16. Johnston-MacAnanny EB, DiLuigi AJ, Engmann LL, Maier DB, Benadiva CA, Nulsen JC. Selection of first in vitro fertilization cycle stimulation protocol for good prognosis patients: gonadotropin releasing hormone antagonist versus agonist protocols.
J Reprod Med 2011;56:12-6.

17. Pu D, Wu J, Liu J. Comparisons of GnRH antagonist versus GnRH agonist protocol in poor ovarian responders undergoing IVF. Hum Reprod 2011;26:2742-9.

18. Huang SY, Huang HY, Yu HT, Wang HS, Chen CK, Lee $C L$, et al. Low-dose $\mathrm{GnRH}$ antagonist protocol is as effective as the long $\mathrm{GnRH}$ agonist protocol in unselected patients undergoing in vitro fertilization and embryo transfer. Taiwan J Obstet Gynecol 2011;50:432-5. 